\author{
Classification \\ Physics Abstracts \\ $7.260-7.820-7.830-7.700$
}

\title{
RAYLEIGH SOUND WAVE PROPAGATION ON A GALLIUM SINGLE CRYSTAL IN A LIQUID He $^{3}$ BATH
}

\author{
G. BELLESSA \\ Laboratoire de Physique des Solides $(*)$ \\ Université Paris-Sud, Centre d'Orsay, 91405 Orsay, France
}

(Reçu le 5 février 1975, accepté le 27 février 1975)

\begin{abstract}
Résumé. - Nous décrivons l'observation de la propagation d'ondes élastiques de Rayleigh sur un monocristal de gallium. Les fréquences d'études sont comprises entre $40 \mathrm{MHz}$ et $120 \mathrm{MHz}$ et la température d'étude la plus basse est $0,4 \mathrm{~K}$. La vitesse et l'atténuation sont étudiées à des températures différentes. L'atténuation des ondes de Rayleigh par le bain d' $\mathrm{He}^{3}$ est aussi étudiée à des températures et des fréquences différentes.
\end{abstract}

Abstract. - We report the observation of the Rayleigh sound wave propagation on a gallium single crystal at frequencies up to $120 \mathrm{MHz}$ and temperatures down to $0.4 \mathrm{~K}$. The velocity and the attenuation are studied at different temperatures. The attenuation of Rayleigh waves by the $\mathrm{He}^{3}$ bath is also studied at different temperatures and frequencies.

Elastic surface waves have been studied extensively on piezoelectric materials, mainly quartz and lithium niobate [1]. There are also some studies of Rayleigh waves on piezoelectric films evaporated on a glass substrate [2]. The effects of thin metallic films evaporated on a piezoelectric substrate have been studied $[3,4]$ and the effects of the electrons on the propagation of Rayleigh waves have been observed (mainly the normal-superconducting transition). However the magnitude of these effects are small, because the thickness of the evaporated films are much smaller than the Rayleigh wavelength. In this letter, we report the observation of the Rayleigh wave propagation on a gallium single crystal. We report some preliminary results about the attenuation variation in the normal-superconducting transition and about the attenuation of Rayleigh waves by liquid $\mathrm{He}^{3}$.

Experimental technique. - The single crystal is made with very pure gallium in a plastic mold which has two polished faces [5]. The single crystal is not machined and good surfaces for the Rayleigh wave propagation are obtained directly by this method. The flat surfaces of the gallium crystal are normal to the $\mathbf{b}$ axis (Fig. 1) and a film of $\mathrm{ZnS}$ is evaporated (about $6000 \AA)$ onto the crystal. This piezoelectric film acts as an electro-mechanical transducer. Two metallic

$\left({ }^{*}\right)$ Laboratoire associé au C.N.R.S. combs are evaporated on the $\mathrm{ZnS}$ film (Fig. 1). The comb teeth are $20 \mu$ wide and are distant from each other of $40 \mu$. Each comb consists of 40 teeth and is distant from its neighbour by $6 \mathrm{~mm}$. A RF pulse (about $0.5 \mu$ s duration) is applied between one comb and the sample (the metallic sample is grounded).

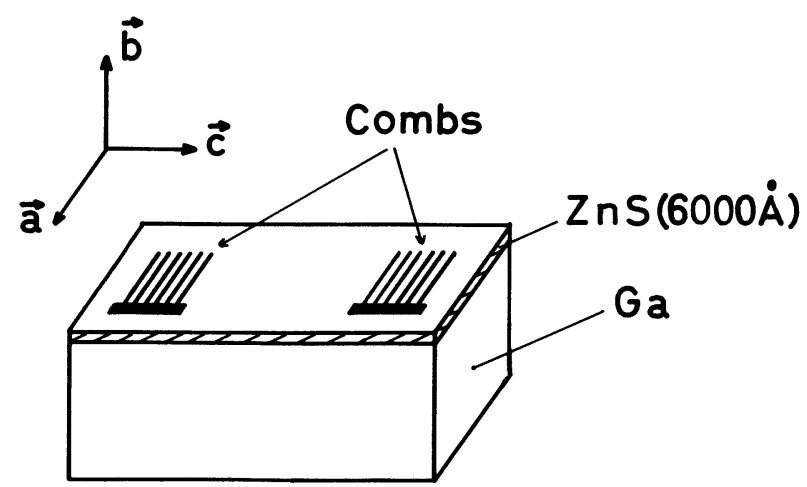

Fig. 1. - Experimental arrangement for the Rayleigh wave generation. The a, b, $\mathbf{c}$ axis are the crystallographic axis of the Gallium crystal with the usual notations.

A Rayleigh wave is generated on the metal surface if the frequency of the RF pulse is such that the wavelength of the Rayleigh wave equals the teeth spacing. The acoustic surface wave propagates normal to the comb teeth and reaches the second comb (which is 
well parallel to the first one) after a certain time. At this time an RF pulse is observed on the second comb. We observe no reflexion of the acoustic pulse on the combs. So there are no successive echoes (as in the case of bulk waves) and it is not possible to make an absolute measurement of the attenuation. In order to do this, it would be necessary to evaporate three metallic combs on the crystal. Figure 2 shows the delayed pulse that we observe on the second comb after amplification and detection. The first saturated pulse corresponds to the pulsed excitation of the emitter comb. We have observed Rayleigh waves on gallium at $40 \mathrm{MHz}$ (fundamental), $80 \mathrm{MHz}$ and $120 \mathrm{MHz}$ (2nd and 3rd harmonics).

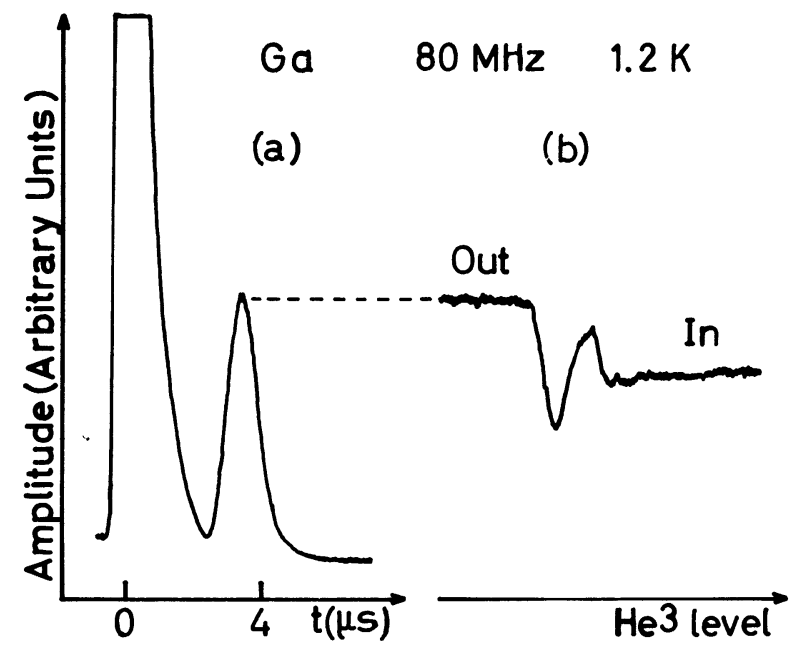

FIG. 2. - a) Recorder tracing of the delayed pulse corresponding to the Rayleigh wave train. The first saturated pulse corresponds to the pulsed excitation of the emitter comb. $b$ ) Recorder tracing of the variation delayed pulse amplitude versus the liquid level. Out means surface sample out of the $\mathrm{He}^{3}$ bath. The liquid level is changed by liquefying $\mathrm{He}^{3}$ gaz slowly at $1.2 \mathrm{~K}$.

Velocity and attenuation measurements. - There are two ways to measure the Rayleigh wave velocity. The first one is to measure the transit time of the pulse between the two combs. The second is to adjust the frequency of the RF pulse in order to obtain the largest amplitude of the delayed pulse on the receiver comb. This last method is the most sensitive because the selectivity of the comb is proportional to the square of the number of teeth and our combs (40 teeth) are very selective [1]. Table I gives the results of our measurements at different temperaturès and the bulk wave velocities for comparison (taken from ref. [6]). As we have written above, we cannot measure the absolute attenuation but we can measure the variation of the attenuation versus temperature. The attenuation is found to be roughly constant between $4.2 \mathrm{~K}$ and $1.1 \mathrm{~K}$ (superconducting transition of $\mathrm{Ga}$ ). Below $1.1 \mathrm{~K}$ the attenuation is very sensitive to the temperature. We have found for the variation of the attenuation of surface waves at $80 \mathrm{MHz}$ :

$$
\alpha_{N}(1.2 \mathrm{~K})-\alpha_{\mathrm{S}}(0.4 \mathrm{~K})=11 \pm 0.5 \mathrm{~dB} / \mathrm{cm} \text {. }
$$

TABLE I

Rayleigh waves velocities (in $10^{5} \mathrm{~cm} / \mathrm{s}$ ) in gallium at different temperatures (in Kelvin). The $\mathbf{b}$ axis is normal to the surface and the propagation is along the caxis. The sound velocities for the bulk wave propagation along the b axis are taken from $K . R$. Lyall and J. F. Cochran [6].

\begin{tabular}{lccc}
\multicolumn{1}{c}{$T$} & 300 & 77 & 4 \\
Rayleigh & - & - & - \\
Longitudinal & $2.26 \pm 0.02$ & $2.38 \pm 0.02$ & $2.40 \pm 0.02$ \\
Shear (vibration // c) & 3.95 & 4.07 & 4.08 \\
Shear (vibration //a) & 2.45 & 2.56 & 2.57 \\
& 2.64 & 2.79 & 2.81
\end{tabular}

We have also put the sample in a magnetic field (normal to the surface). At $0.4 \mathrm{~K}$ there is a sharp change in the attenuation in the vicinity of the superconducting transition (around $60 \mathrm{Oe}$ ). In the normal state, the attenuation increases up to $2 \mathrm{kOe}$. Then it remains roughly constant up to $10 \mathrm{kOe}$. The total variation of the attenuation at $0.4 \mathrm{~K}$ and $80 \mathrm{MHz}$ is found to be :

$$
\alpha_{\mathrm{N}}(H=10 \mathrm{kOe})-\alpha_{\mathrm{S}}(H=0)=20 \pm 1 \mathrm{~dB} / \mathrm{cm} .
$$

Attenuation of rayleigh waves by liquid $\mathrm{He}^{3}$. The main mechanism of absorption of Rayleigh waves by ambient media is the emission of compressional waves in the fluid during the propagation [1]. This process gives for the attenuation :

$$
\alpha=10\left(\log _{10} \text { e) } \frac{\rho_{\mathrm{F}} V_{\mathrm{F}}}{\rho V_{\mathrm{R}}} \frac{1}{\lambda_{\mathrm{R}}} \mathrm{dB} / \mathrm{cm}\right.
$$

where $\rho_{\mathrm{F}}$ is the fluid density, $V_{\mathrm{F}}$ the sound velocity in the fluid, $\rho$ the density of the solid, $V_{\mathrm{R}}$ and $\lambda_{\mathrm{R}}$ the velocity and the wavelength of the Rayleigh wave. Experimentally, we start from a sample surface out of the $\mathrm{He}^{3}$ bath and we record the amplitude of the delayed pulse corresponding to the Rayleigh wave train (Fig. 2). We then change the liquid level in the cryostat and we record a decrease of the delayed pulse amplitude as soon as liquid $\mathrm{He}^{3}$ covers the sample surface. Figure 2 shows such a recorder tracing. As can be seen on the curve, the attenuation does not fall directly to its final value. We shall consider this point later. We have measured the attenuation variation for different temperatures and frequencies. In order to measure the attenuation variation, we do not take simply the ratio of the pulse heights in the two cases. This method can introduce errors owing to the possible non-linearity of the amplifiers. We avoid this difficulty, by changing the RF generator amplitude in order to obtain the same pulse height in the two cases. We then take the ratio of the corresponding values of the generator amplitude. Our results are reported in Table II. There is no measurement at $120 \mathrm{MHz}$ and $1.2 \mathrm{~K}$ because the attenuation of Rayleigh waves in gallium is very large above the 


\section{TABLE II}

Measured values of the attenuation (in $\mathrm{dB} / \mathrm{cm}$ ) of Rayleigh waves in gallium by liquid $\mathrm{He}^{3} . \alpha_{\mathrm{cal}}$ is the calculated value obtained from eq. 1. At $0.4 \mathrm{~K}$ Gallium is superconducting. $T$ is the temperature and $F$ the frequency of the Rayleigh wave.

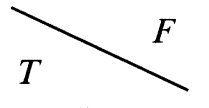

$1.2 \mathrm{~K}$

$0.4 \mathrm{~K}$

$\alpha_{\text {cal }}$
$40 \mathrm{MHz}$

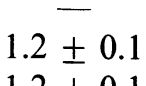

$1.2 \pm 0.1$

0.77

\section{$80 \mathrm{MHz}$}

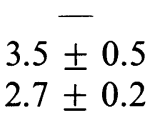

1.54

$4 \pm 0.5$
$120 \mathrm{MHz}$

superconducting transition temperature and it has not been possible to obtain a significant value. $\alpha_{\text {cal }}$ is the calculated value obtained from eq. (1). The calculated values are systematically smaller than the experimental ones. It therefore seems there is attenuation mechanism other than that due to the emission of compressional waves during the propagation. It is not necessary to consider in the present case the attenuation process arising from frictional losses [1]. This mechanism gives indeed a negligible attenuation equal to $5 \times 10^{-3} \mathrm{~dB} / \mathrm{cm}$ at $80 \mathrm{MHz}$. At $0.4 \mathrm{~K}$ ( $\mathrm{Ga}$ is then superconducting), the attenuation by liquid $\mathrm{He}^{3}$ versus frequency does not deviate appreciably from a linear law in agreement with eq. (1). At $1.2 \mathrm{~K}$ the attenuation is no longer proportional to the frequency. Perhaps, what we are observing here is, at its beginning, the effect of the electrons on the surface wave attenuation by liquid helium. This effect is involved in the Kapitza resistance problem [7] and has been observed in gallium [8]. Expériments at higher frequencies are necessary to elucidate this interesting point.
In conclusion let us consider the attenuation variation just as the liquid covers the sample surface (Fig. 2). In this case the attenuation curve has successively a maximum and a minimum before reaching its final value (in Fig. 2 the amplitude minimum corresponds to the attenuation maximum). This behaviour can be explained by taking into account the effect of an helium film on the sample surface. The attenuation process leading to eq. (1) does not consider any return of the emitted compressional waves on the surface sample. If, there is a helium film on the sample, instead of a semi-infinite media, it is then necessary to consider such reflections. Schematically the Rayleigh wave radiates a compressional wave which is almost normal to the surface (the angle is equal to Arc $\sin V_{\mathrm{F}} / V_{\mathrm{R}}=4^{\circ}$ ). The compressional wave is reflected on the upper surface of the film and then returns toward the sample surface. There are therefore interferences between the Rayleigh wave and the reflected compressional wave. If the interferences are destructive i.e. if $2 d \cong(2 n+1) \lambda_{\mathrm{F}} / 2$ (where $d$ is the film thickness and $\lambda_{\mathrm{F}}$ the wavelength in the fluid), there is an attenuation maximum. There is also an attenuation minimum if $2 d \cong n \lambda_{\mathrm{F}}$. Thus the maximum and the minimum of the attenuation in figure 2 would correspond to a film thickness of $\lambda_{F} / 4=0.5 \mu$ and $\lambda_{\mathrm{F}} / 2=1 \mu$ respectively. Experimentally, if the film thickness was well defined there would be absorption resonances for many thicknesses and these resonances would be very sharp. In our case the film thickness is probably not well defined and we observe only a very damped resonance.

The author is indebted to L. Dumoulin and M. Boix for useful advices about the evaporation techniques and to J. Joffrin for a fruitful discussion.

\section{References}

[1] Dransfeld, K. and Salzmann, E., Physical Acoustics, edited by W. P. Mason (Academic, New York) 1970, vol. 7, p. 219.

[2] Inaba, R., Kajimura, K. and Mikoshiba, N., J. Appl. Phys. 44 (1973) 2495.

[3] AKaO, F., Phys. Lett. 30A (1969) 409.

[4] Krätzig, E., Phys. Rev. B 7 (1973) 119.

[5] The author is indebted to P. DE LA BRETÈQue of Alusuisse
- France S. A. for the supply of very pure gallium

[6] Lyall, K. R. and Cochran, J. F., Can. J. Phys. 49 (1971) 1076. [7] Andreev, A. F., Zh. Eksp. Teor. Fiz. 43 (1962) 1535 (Sov. Phys. JETP 16 (1963) 1084).

[8] Wagner, F., Kollarits, F. J. and Yaqub, M., Phys. Rev. Lett. 32 (1974) 1117. 